\title{
The reproducibility of manual and fixed tensioning in anterior cruciate ligament reconstruction
}

\author{
Richard Stevens ${ }^{1}$ \\ Jeevan Chandrasenan ${ }^{2}$ \\ Fazal Ali ${ }^{2}$ \\ Paul Haslam 4 \\ Nicolas Nicolaou ${ }^{3}$
}

\author{
${ }^{1}$ Northern General Hospital, Sheffield, UK \\ ${ }^{2}$ Chesterfield Royal Hospital, Chesterfield, UK \\ ${ }^{3}$ Sheffield Children's Hospital, Sheffield, UK \\ ${ }^{4}$ Doncaster Royal Infirmary, Doncaster, UK
}

Corresponding author

Richard Stevens

Northern General Hospital,

Sheffield, UK

E-mail:mda03rjs@doctors.org.uk

\section{Summary}

Introduction: Tensioning of anterior cruciate ligament grafts is required to restore knee stability. No consensus has been achieved as to what is the best tension. Little work has been done as to whether the most common method of tensioning, by manually pulling, is reproducible.

Methods: A model was developed that allowed cycling \& tensioning of a graft while measuring tension in it. Five surgeons were asked to repeatedly tension the graft as they would in theatre. The same was done using a fixed tensioning device. The coefficient of variability (CV) was calculated for the final tensions.

Results: The average CV for the surgeons tensioning was $27 \%$ (19 to $41 \%$ ). Fixed tensioning produced a CV of $9 \%$. Welch ANOVA testing demonstrated a significant inter-surgeon variability $(p<0.0001)$. Unpaired T-tests comparing each surgeon to the tensioner demonstrated a significant difference in tensions $(p<0.05)$ in all results.

Conclusions: A CV less than $10 \%$ is considered evidence that a method is reliable \& therefore this study provides further evidence that manual tensioning is not a reproducible way of applying tension to a graft. Fixed tensioning is reproducible but the clinical impact of this needs further study. Level of evidence: IIIb.

KEY WORDS: ACL, cruciate, tension.

\author{
Introduction
}

Anterior cruciate ligament reconstruction is a common procedure in a young, athletic population. The indications cited for reconstruction, and the subsequent intensive rehabilitation, are to allow return to function and prevent secondary degenerative changes. The success of the procedure is reliant on a number of biological and mechanical factors.

Biological factors include post-operative hemorrhage, synovitis, cytokine levels, cell infiltration and revascularisation of the graft ${ }^{1}$.

Mechanical factors include graft selection, graft condition, position of bone tunnels, fixation method, angle of flexion during fixation, post-operative rehabilitation, preconditioning of the graft and initial tension of the graft ${ }^{2}$. While a consensus has been reached on the majority of these, there is still controversy regarding the ideal initial tension required to restore stability to the knee.

Numerous studies have looked at the effect of different tensions on the outcome of $\mathrm{ACL}$ reconstruction. Some argue a relatively low tension is required to restore normal laxity and too high a tension over tightens the knee, leading to the tibia subluxing posteriorly, abnormal articulation, and cartilage degradation $^{1,3,4}$. Others argue this is not the case, or if so is transient, and a higher tension is required due to early creep in the graft leading to a loss of tension, and increase laxity $2,5-8$. The only systematic review looking at the topic concluded that the variation in methodologies of the studies done, was so much so, and the quality of the randomised control trials so low, that no meaningful statistical testing was possible ${ }^{9}$.

Despite significant work trying to identify the optimum initial tension, very little work has been done to find out if it is possible to accurately reproduce a tension manually, as is most commonly practiced. One study has looked at variability between repeated efforts by the same surgeon, showing a range of coefficients of variation $(\mathrm{CV})$ of $16-30 \%{ }^{10}$. While there is no definite standard, a CV of less than $10 \%$ is considered an acceptable level of variability in other scientific fields ${ }^{11}$. Their methodology had limitations and we hypothesise that manual tensioning while cycling the knee may be more variable. There are fixed tensioning devices available commercially which preset and maintain a tension, however we could not find any studies looking at their reproducibility or comparing them to 
manual tensioning. Therefore, our second hypothesis is that the tension applied to a graft is less variable using these.

\section{Material and methods}

A model to replicate a left lower limb was constructed that allowed cycling of the limb and tensioning of a graft as would be done intra-operatively. The aim was to allow repeated use by different users without changing any variables other than the user. The model was constructed from wood using anthropometric measurements, with a hinge joint representing the knee, and a hinge and pivot for the hip. Replica femoral and tibial tunnels were drilled, with the tibial tunnel placed as it would be intra-operatively. 4 strands of No. 5 Ethibond (Ethicon, USA) graft were passed through the tunnels and attached to a force gauge (PCE-HS 150N, PCE instruments, UK) anchored to the femoral side. A video camera (GoPro, Hero 3) was attached to record the readings with the screen not visible to the user. The stuffing and foam skin from a knee injection model (Sawbones, USA) were then used to replicate the soft tissues, in particular where the user may wish to rest their hand on the anterior tibia while tensioning the graft (Fig. 1). The model allowed flexion and extension at the knee and circumduction at the hip.

The research was conducted following review of international ethical standards, and deemed not to require ethics committee approval before commencing. Every effort was made to adhere to the principles and recommendations of this Journal, as set out by Padulo et al. ${ }^{12}$.

Preliminary testing was performed on the model to try to replicate the tension-flexion curve that the normal ACL goes through when moving the knee ${ }^{13}$. We found that our initial femoral tunnel placement didn't achieve this therefore a number of different positions were tried until a combination was achieved that came close to mimicking this curve (Fig. 2).

Four soft tissue knee surgeons and one trainee were then asked to manually tension the graft and cycle the model as they would intra-operatively. Each was asked to do this 10 times over the course of a few days with a break of at least 90 minutes between goes. 10 cycles in a similar fashion were then done
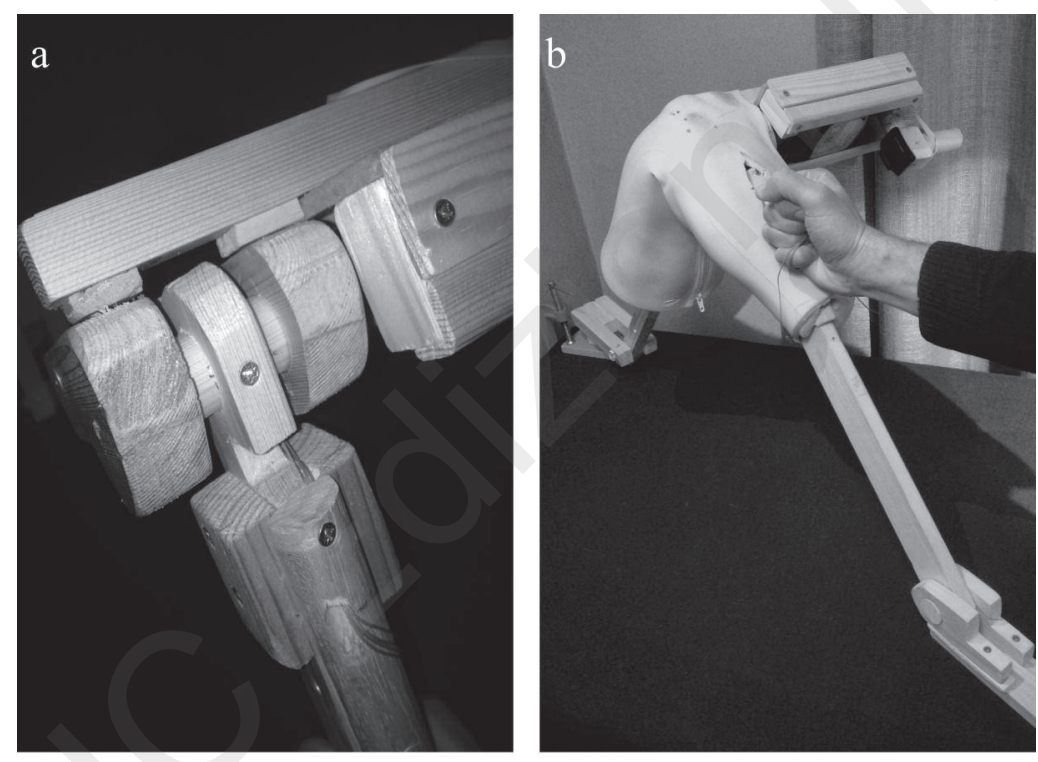

Figure 1. Images showing the model. Clockwise from left: a) constrained hinge representing the knee with sutures visible entering the tibial tunnel (white arrow); b) model with user's hand tensioning the graft \& force gauge visible on far side; c) model in extension.

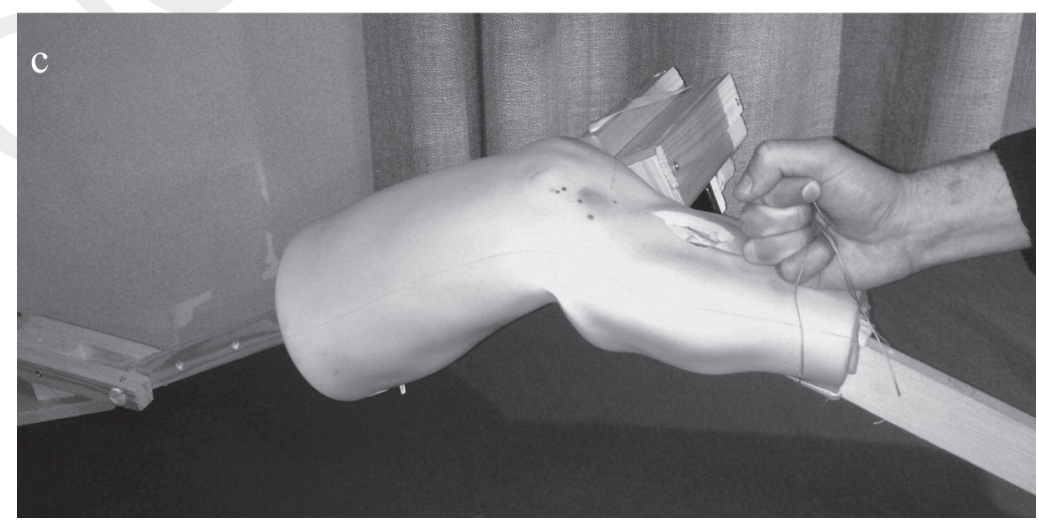




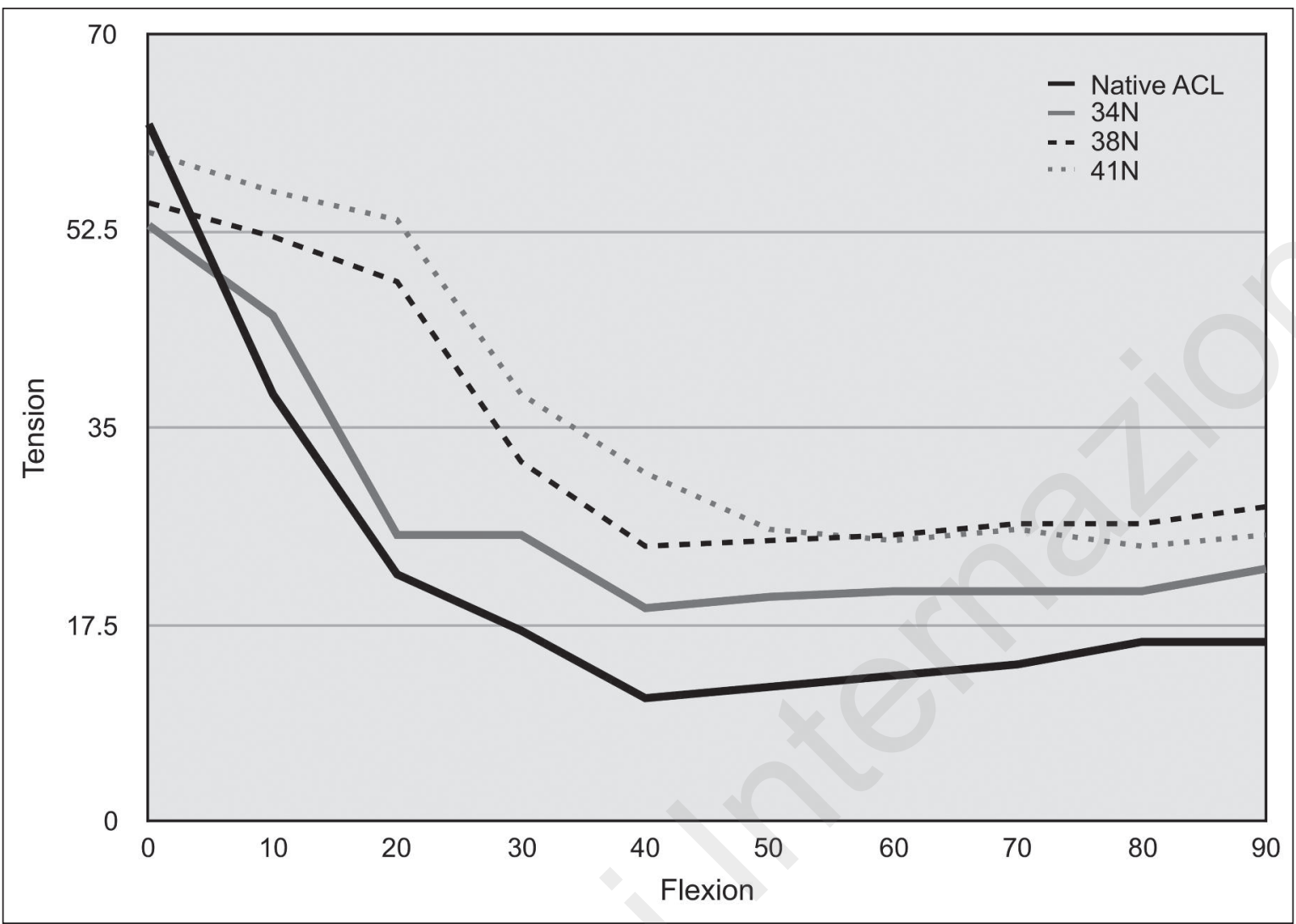

Figure 2. Final model tension-flexion curves at different initial tensions (and compared to native ACL).

using the fixed tension device (TunneLoc, Zimmer Biomet, UK), tensioned to the $50 \mathrm{~N}$ mark. During each attempt, a recording of the display showing the force on the graft (in Newton's) was taken and data was imputed into Microsoft Excel on watching the recordings back. The coefficients of variability for the final tensions were calculated for each surgeon. Preliminary analysis of data indicated a lack of homogeneity of variance therefore a Welch ANOVA test was used to see if differences in final tension between surgeons was significant with Post-Hoc testing using Games-Howell tests. Unpaired t-tests were used to compare each surgeon's mean final tension to the fixed tensioning results.

\section{Results}

Each surgeon had 10 attempts on the model, however some efforts were discarded on watching back the recordings of the force gauge screen due to glare. This meant there were 8 useable attempts for Surgeon A, 10 for Surgeon B, 8 for Surgeon C, 6 for Surgeon $D, 7$ for Surgeon $E$, and 10 using the fixed tensioning device.

The Coefficients of variability (CV) for the final tension on the graft for each surgeon were 21, 41, 28, 28, 19 and $9 \%$ for the fixed tensioner (Fig. 3).
Figures 4 and 5 demonstrate the mean final tensions (in Newtons) for each user with the standard error bars \& outliers plotted. Variability in the final tension values between the five surgeons was assessed for statistical significance using a Welch ANOVA test and found to be statistically significant $\left(p=0.006 \times 10^{-6}\right)$.

Each surgeon's final tensions were compared to the fixed tensioning device using an unpaired T-test. All surgeons demonstrated a statistically significant difference $(p<0.05)$ in final tension value.

\section{Discussion}

The aim of this study was to test the hypothesis that there is significant variation in final tension values when ACL grafts are tensioned manually. Leading on from this, our second hypothesis was that variation would be improved by using a fixed tensioning device (TunneLoc, Zimmer Biomet). Our results confirm both these hypotheses, with each surgeon having a CV $>10 \%$ (range $19-41 \%$ ), while the fixed tensioning device had a CV of $9 \%$.

The strengths of this study are that it allowed for testing on a model that limited the variables as much as possible; allowed for extended gaps between repeat attempts to reduce time dependent measurement bias; and compared manual tensioning with an alter- 


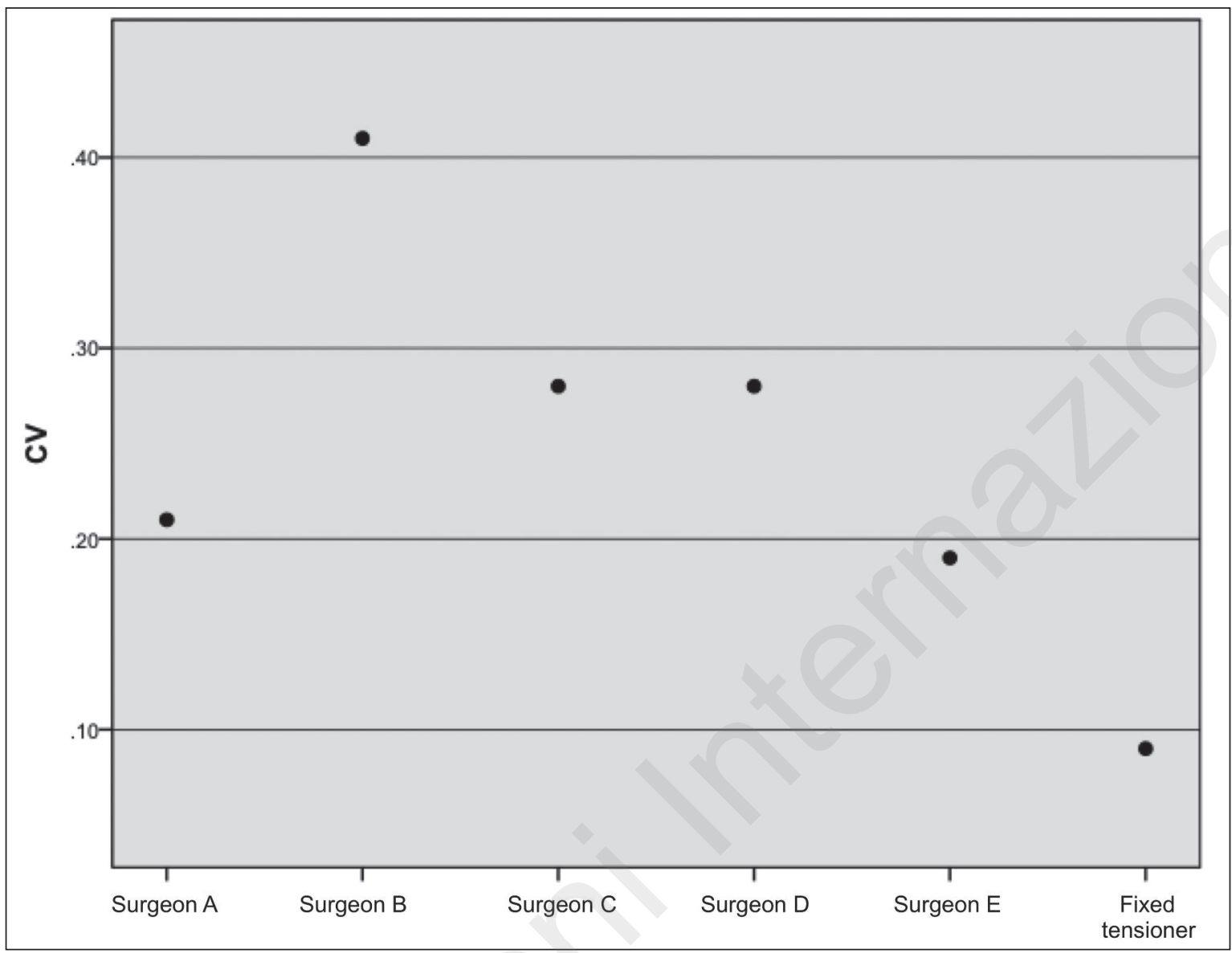

Figure 3. Coefficients of variability (CV) for each surgeon.

\begin{tabular}{|l|r|r|r|r|r|r|}
\hline & Surgeon A & Surgeon B & Surgeon C & Surgeon D & Surgeon E & TunneLoc \\
\hline Mean Final tension & 16.63 & 25.70 & 18.25 & 12.92 & 69.40 & 33.50 \\
\hline Standard deviation & 4.15 & 9.44 & 5.15 & 3.46 & 12.15 & 3.03 \\
\hline
\end{tabular}

Figure 4. Mean final tension in graft $(\mathrm{N})$ for each user.

native, fixed tensioning method. These steps in the methodology, and the increased number of recordings taken, are improvements on the small number of studies that already exist in this area.

This study has limitations. The model used cannot perfectly reproduce the movement and feel of a lower limb. Efforts were made to maximise our models realism with a number of prototypes being made. The final model was selected after testing by the lead surgeon. Synthetic skin and foam from a knee injection model were used to replicate the soft tissue component, but the underlying 'knee joint' was a constrained hinge. Preliminary testing with a fixed tension applied with the knee in 30 degrees of flexion was performed, the knee cycled and the tension recorded to produce a tension-flexion curve. This has previously been described for the native $\mathrm{ACL}^{13}$ and we modified our model to try to replicate this curve.
This was achieved by having two femoral tunnels, with two strands of Ethilon in each, before attaching to the force gauge. Other alternatives were also considered. A cadaveric limb would not feel or move the same as intraoperatively and the same is true for animal models. Plastic bone models were too underconstrained. Therefore, while the model is not a perfect replica, one of its strengths is that it allowed for a similarly complex manual task to be performed with limited variables and recordable results.

Other limitations include a small number of surgeons and attempts by each. We did get more efforts per surgeon than the previous similar study but the nature of ACL reconstruction and the multiple variables that are implicated in its success means it is difficult to adequately power any study looking at just one of these factors, and its effect on a successful outcome. One of our surgeons (Surgeon E) had a far higher 


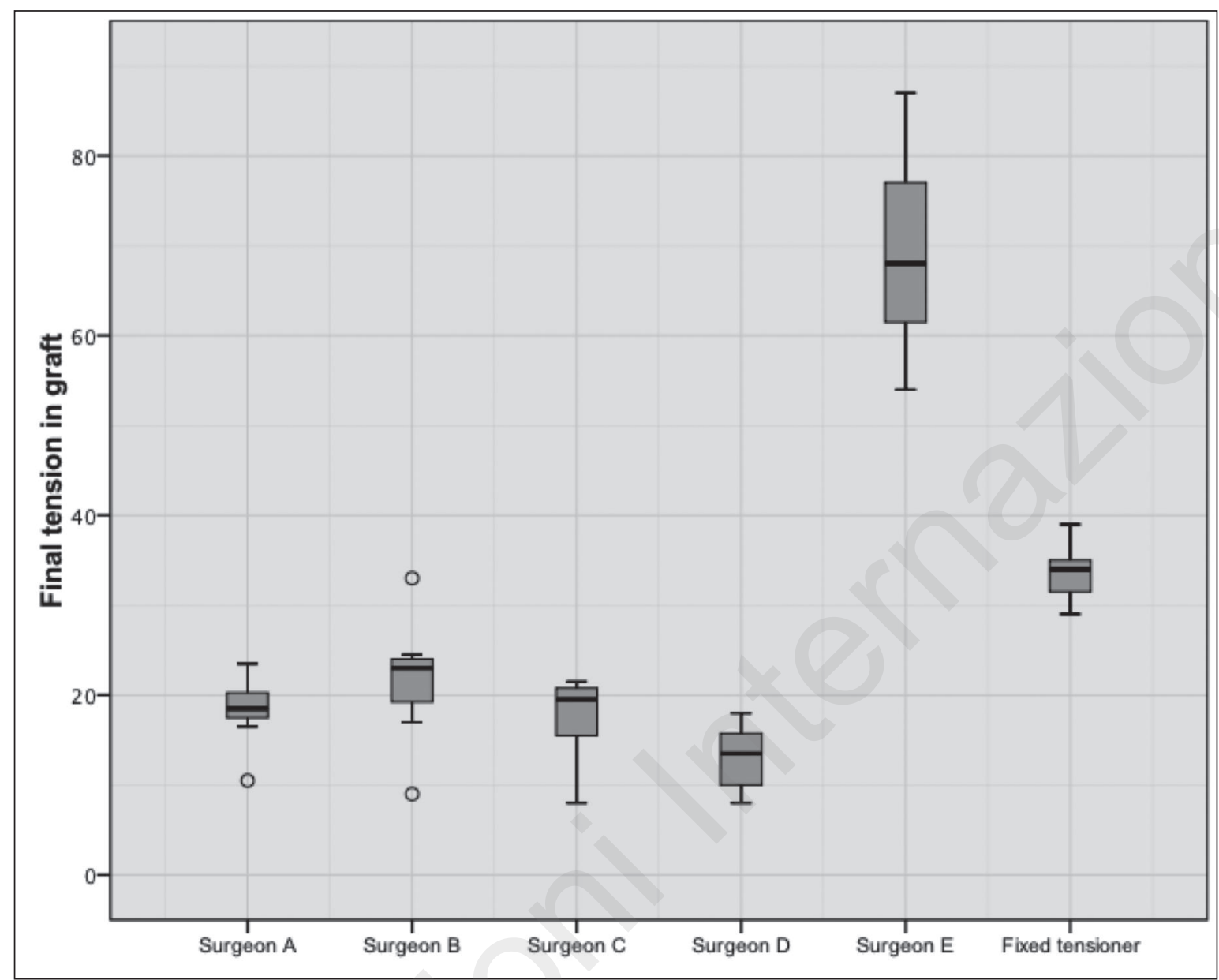

Figure 5. Boxplot of final tensions for each surgeon.

mean tension than the others. The only explanation we have for this is that he secured the suture by clamping in a Kocher clip then wrapping it around this \& pulling on the clip. We hypothesise that this allowed him to pull harder \& tension more as his grip was improved. Although the primary aim of this study was not to look at the absolute values of tension applied, it is worth noting these as they have an impact on graft degeneration. Animal studies have shown that under tensioning or over tensioning the ACL graft can lead to intra-substance degeneration and a reduction in mechanical strength ${ }^{14,15}$. A stress free ligament or tendon is known to degrade, but none of our surgeons finished with a tension free graft at the end of cycling. Rat models ${ }^{14}$ suggest detrimental histological changes when a graft is over-tensioned only 5 newtons more than their ideal tension of 5 newtons. In dog models ${ }^{15}$, similar histological changes were seen when higher tensions were applied (20 newtons). These animal models suggest that it is possible to over-tension a graft, increasing the tension only slightly from an optimum value. The optimum tension in humans is still unknown. Although it is likely to be higher than these, the variability demonstrated by manual tensioning in our study may mean a proportion of patients are being exposed to suboptimal tensions that could be avoided with fixed tensioning, if the optimum tension was known.

We found very little previous work on intra- or intersurgeon variability in the literature. A randomised control trial by Nicholas et al. ${ }^{7}$ mentioned a pilot study they performed looking at the tensions applied by 3 surgeons, ten times each using bone-patella-bone grafts, and documented CVs of $9.1,18.1$ and $31.1 \%$.

Only one study, O'Neil et al. ${ }^{10}$, set to look specifically at reproducibility of manual tensioning. They tested 6 surgeons, five times each, in quick succession. They demonstrated a range of $\mathrm{CV}$ of $16-33 \%$ and concluded that manual tensioning does not produce consistent results, using a $\mathrm{CV}$ of $<10 \%$ to represent reliable reproducibility. We used the same cut-off in this study. Our findings support these previous studies in demonstrating that manual tensioning does not produce consistent results but we have demonstrated even poorer reproducibility. It is hard to compare our methodology to Nicholas et al. ${ }^{7}$, and explain why our results are different, because their pilot study methodology is not described in detail. O'Neil et al. ${ }^{10}$ 
used a plastic bone model and porcine grafts. The model allowed flexion and extension of the knee but no replica hip joint was included. In our practice, the graft is tensioned and the knee cycled through flexion and extension with the patient supine. To do this requires some circumduction at the hip. Our model allowed this by having a pivot and hinge to replicate the hip, and we suggest that this more complex manual task may partly explain the higher $\mathrm{CV}$.

Another possible explanation is our choice of graft. Rather than use porcine or cadaveric grafts, we chose to simulate the graft using 4 strands of No.5 Ethibond (Ethicon, USA). We decided on this because it allowed repeated use of the same graft. Using animal or cadaveric grafts would require multiple grafts with different sizes, grafts being thawed and refrigerated, and repeatedly tensioned - all affecting the mechanical properties. We felt this introduced too many variables. However, using the Ethilon meant that if the pulling force was reduced, the strands could easily slide in the tunnels, reducing the tension reading, unlike with a real graft which has a diameter matched to the diameter of the tunnels and therefore may 'catch'. This may be a contributing factor to our results.

While this study has demonstrated that variability in manual tensioning is higher than previously documented for manual tensioning, it does not address if this makes a clinical difference. The final tensions recorded were taken and then the tension released rather than being definitively fixed to the tibia. For manual tensioning, we use an interference screw, and it is likely that the tension would increase further as the screw is advanced and the graft material pulled further. The fixed tensioning device secures the graft with a plug and it is unlikely that this increases tension further as there is no screw mechanics at play. We could not simulate the bone-fixation device interface with our model to test this.

We conclude that there is poor reproducibility of manual tensioning in ACL reconstruction but this can be overcome by using a fixed tensioning device. Further work needs to be done to clarify if this makes a clinical difference. While a consensus has not been reached on an ideal tension to place on an ACL graft, this study suggests any further work looking into this area uses a fixed tensioning device to produce reproducible results and allow confident analysis and comparison of data sets.

\section{Conflicts of interest}

None.

\section{Acknowledgements}

No funding was sought for this project. Ethical Committee approval not required.

\section{References}

1. Tohyama H, Yasuda K. Significance of graft tension in anterior cruciate ligament reconstruction. Basic background and clinical outcome. Knee Surg Sports Traumatol Arthrosc. 1998;6:S30-37.

2. Labs K, Perka C, Schneider F. The biological and biomechanical effect of different graft tensioning in anterior cruciate ligament reconstruction: an experimental study. Arch Orthop Trauma Surg. 2002 May;122(4):193-199.

3. Burks R, Leland R. Determination of graft tension before fixation in anterior cruciate ligament reconstruction. Arthroscopy. 1988;4:260-266. Doi: 10.1016/S0749-8063(88)80041-0.

4. Mae T, Shino K, Nakata K, Toritsuka Y, Otsubo H, Fujie H. Optimization of graft fixation at the time of anterior cruciate ligament reconstruction. Part II: effect of knee flexion angle. Am J Sports Med. 2008 Jun;36(6):1094-1100.

5. Kim SG, Kurosawa H, Sakuraba H, lkeda H, Takazawa S. The effect of initial graft tension on postoperative clinical outcome in anterior cruciate ligament reconstruction with semitendinosus tendon. Arch Orthop Trauma Surg. 2006;126:260-264.

6. Numazaki H, Tohyama H, Nakano H, Kikuchi S, Yasuda K. The effect of initial graft tension in anterior cruciate ligament reconstruction on the mechanical behaviours of the femur-grafttibia complex during cyclic loading. Am J Sports Med. 2002;30:800-805.

7. Nicholas SJ, D’Amato MJ, Mullaney MJ, Tyler TF, Kolstad K, McHugh MP. A prospectively randomized double-blind on the effect of initial graft tension on knee stability after anterior cruciate ligament reconstruction. Am J Sports Med. 2004;32: 1881-1886.

8. Boylan D, Greis P, West J, Bachus K, Burks R. Effects of initial graft tension on knee stability after anterior cruciate ligament reconstruction using hamstring tendons; A cadaveric study. Arthroscopy. 2003;19:700-705.

9. Arneja S, McConkey MO, Mulpuri K, Chin P, Gilbart MK, Regan WD, Leith JM. Graft tensioning in anterior cruciate ligament reconstruction: a systematic review of randomized controlled trials. Arthroscopy. 2009 Feb;25(2):200-207.

10. O'Neill BJ, Byrne FJ, Hirpara KM, Brennan WF, McHugh PE, Curtin W. Anterior cruciate ligament graft tensioning. Is the maximal sustained one-handed pull technique reproducible? BMC Research Notes. 2011;4:244.

11. Albert $A$, Zhang $L$. A novel definition of the multivariate coefficient of variation. Biom J. 2010 Oct;52(5):667-675.

12. Padulo J, Oliva F, Frizziero A, Maffulli N. Muscles, Ligaments and Tendons Journal - Basic principles and recommendations in clinical and field science research: 2016 update. MLTJ. 2016;6(1):1-5.

13. Fleming B, Beynnon BD, Johnson RJ, McLeod WD, Pope MH. Isometric versus tension measurements. Am J Sports Med. 1993 Jan;21(1):82-88.

14. Ma R, Schaer M, Chen T, Sisto M, Ying L, Deng X, Rodeo SA. The Initial Anterior Cruciate Ligament Graft Pre-Tension Force Affects Graft Healing: An Experience with a Small Animal Reconstruction Model. Poster presented at: Orthopaedic Research Society Annual meeting; 2014 March 15-18th; New Orleans, USA.

15. Tohyama H, Yasuda K. Significance of graft tension in anterior cruciate ligament reconstruction: basic background and clinical outcome. Knee Surg Sports Traumatol Arthrosc. 1998;6(Suppl 1):S30-37. 